\title{
Transient accumulation, bidirectional movement and tip release of KIF13B in primary cilia
}

Alice Dupont Juhl ${ }^{1,5}$, Zeinab Anvarian ${ }^{2,5}$, Julia Berges ${ }^{2,3,4}$, Daniel Wüstner ${ }^{1, *}$, Lotte B. $\operatorname{Pedersen}^{2, *}$

${ }^{1}$ Department of Biochemistry and Molecular Biology, University of Southern Denmark, Campusvej 55, DK-5230 Odense M, Denmark

${ }^{2}$ Department of Biology, University of Copenhagen, Universitetsparken 13, DK-2100

Copenhagen $\varnothing$, Denmark

${ }^{3}$ Department of Biomedicine, Facultad Ciencias Experimentales, Universidad Francisco de

Vitoria, Ctra. Pozuelo-Majadahonda Km. 1.800, 28223 Pozuelo de Alarcón (Madrid), Spain

${ }^{4}$ Present address: Universidad Autónoma de Madrid, Facultad de Medicina, C/Arzobispo

Morcillo, 4, 28029, Madrid, Spain

${ }^{5}$ These authors contributed equally

*Co-corresponding authors: wuestner@bmb.sdu.dk; lbpedersen@bio.ku.dk

Further information and requests for resources and reagents should be directed the lead contact Lotte B. Pedersen (lbpedersen@bio.ku.dk) 


\begin{abstract}
Primary cilia are microtubule-based sensory organelles whose assembly and function rely on the conserved bidirectional intraflagellar transport (IFT) system, which is powered by anterograde kinesin-2 and retrograde cytoplasmic dynein 2 motors. Nematodes additionally employ a male-specific kinesin-3 motor, KLP-6, which regulates ciliary content and function by promoting release of bioactive extracellular vesicles (EVs) from cilia. Here we provide evidence that a KLP-6 homolog, KIF13B, undergoes bursts of bidirectional movement within primary cilia of cultured mammalian cells, as well as occasional EV-like release from the ciliary tip. KIF13B intraciliary movement and tip release required its own motor domain, and a ciliary membrane marker, SMO-tRFP, was not co-released with KIF13B from cilia. Our work provides the first demonstration of intraciliary movement by a vertebrate kinesin other than IFT kinesin-2 motors and suggests that KIF13B regulates ciliary membrane content by promoting EV release at specific ciliary membrane subdomains.
\end{abstract}




\section{Introduction}

Primary cilia are microtubule-based sensory organelles found on the surface of almost all animal cells and are pivotal for regulating diverse signaling pathways such as Sonic hedgehog (Shh) and polycystin-1/polycystin-2-mediated signaling (Anvarian et al., 2019). Cilia are compartmentalized organelles whose assembly, maintenance and function depend on the conserved intraflagellar transport (IFT) machinery that via kinesin-2 and cytoplasmic dynein 2 motors, respectively, brings IFT-A and IFT-B complexes with associated ciliary cargoes into and out of the organelle (Pedersen and Rosenbaum, 2008, Taschner and Lorentzen, 2016). Ciliary composition and function are additionally regulated by the transition zone at the ciliary base (Garcia-Gonzalo and Reiter, 2017), as well as by shedding and environmental release of extracellular vesicles (EVs) from cilia (Wood and Rosenbaum, 2015, Akella and Barr, 2021). Such EVs act as signal-carrying entities in e.g. Caenorhabditis elegans and Chlamydomonas (Wang et al., 2014, Wood et al., 2013, Luxmi et al., 2019) and may rid primary cilia of unwanted material in vertebrates (Nager et al., 2017, Phua et al., 2017). The precise mechanisms underlying the release of EVs from cilia are not clear, but the process is negatively regulated by the BBSome, a retrograde IFT membrane cargo adaptor (Lechtreck, 2015), in both mammalian cells (Nager et al., 2017) and C. elegans (Akella et al., 2020). In mammalian cells, release of EVs from cilia requires local polymerization of actin at the EV release site (Nager et al., 2017), whereas in C. elegans the kinesin-3 motor KLP-6 was shown to move anterogradely within male-specific sensory cilia (Morsci and Barr, 2011), where it promotes ciliary targeting and release of polycystin-2-carrying EVs to control male mating behavior (Wang et al., 2014). Several other factors regulating EV biogenesis, shedding and release from cilia in C. elegans have been identified, many of which affect axonemal tubulin post translational modification and hence KLP-6 motility within cilia (Akella and Barr, 2021). It is unknown if vertebrate kinesin-3 motors similarly move within primary cilia and promote ciliary EV release, and to date KLP-6 is the only kinesin apart from IFT kinesin-2 motors with demonstrated intraciliary motility.

We previously showed that a KLP-6 homolog, KIF13B, localizes to primary cilia in mouse fibroblasts and immortalized human retinal pigment epithelial (hTERT-RPE1) cells, and that its depletion in these cells leads to ciliary accumulation of the cholesterol-binding membrane protein CAV1 as well as impaired Shh signaling (Schou et al., 2017). However, the molecular basis for this phenotype was not clear and it is unknown if KIF13B actually moves within cilia. Here, we show by live cell imaging combined with image analysis and 
simulations that KIF13B undergoes bursts of IFT-like bidirectional movement within primary cilia of hTERT-RPE1 cells. Moreover, we find that KIF13B is occasionally released from the ciliary tip in EV-like particles. Intraciliary movement and ciliary tip release required KIF13B's own motor domain, and a ciliary membrane marker, SMO-tRFP, was not coreleased with KIF13B from cilia in these cells. Our results provide the first demonstration of intraciliary movement by a vertebrate kinesin other than IFT kinesin-2 motors and indicate that KIF13B may regulate ciliary membrane content by promoting ciliary EV release at specific ciliary membrane subdomains.

\section{Results and discussion}

To investigate if KIF13B moves inside cilia we expressed previously validated eGFPKIF13B fusion protein (Schou et al., 2017, Serra-Marques et al., 2020) in hTERT-RPE1 cells stably expressing a red fluorescent ciliary membrane marker, SMO-tRFP (Lu et al., 2015), and subjected cells to serum deprivation to induce ciliogenesis prior to analysis by confocal live cell imaging (see Materials and methods for details). In all cells examined ( $n=59)$, eGFPKIF13B was strongly concentrated at the ciliary base, and 17 of these additionally displayed rapid and transient accumulation of GFP-KIF13B within the cilium itself. Furthermore, in 5 of these 17 cells eGFP-KIF13B underwent EV-like release from the ciliary tip (Figure 1A-E; Movie 1,2). Notably, ciliary SMO-tRFP was not co-released with eGFP-KIF13B under these conditions (Figure 1A; Movie 1,2), indicating that the observed release of eGFP-KIF13B is not due to general disintegration of the ciliary tip. Kymograph analysis indicated that eGFPKIF13B moves bidirectionally within cilia at $0.64 \pm 0.07 \mu \mathrm{m} / \mathrm{s}$ in the anterograde direction (mean \pm sem of $n=37$ measurements from 5 cells) and $0.39 \pm 0.06 \mu \mathrm{m} / \mathrm{s}$ in the retrograde direction (mean \pm sem of $n=36$ measurements from 5 cells). This is within the range of speeds reported for anterograde and retrograde IFT in mammalian cells (Williams et al., 2014), but slower than the reported anterograde speed for KIF13B moving on cytoplasmic microtubules in e.g. HeLa cells (2-3 $\mu \mathrm{m} / \mathrm{s}$; (Serra-Marques et al., 2020)). Kymographs indicated that eGFP-KIF13B moves progressively in both directions without slowing or acceleration and without prolonged pauses at the ciliary tip. From the cumulative histogram of velocities, we find that anterograde movement is slightly faster than retrograde movement (Figure $1 \mathrm{~F}$ and $\mathrm{G}$ ). The cumulative histogram of velocities, $v$, was fitted by a Weibull distribution function of the form $y(v)=1-\exp \left[-(\alpha \cdot v)^{\beta}\right]$ with $\alpha=1.58$ and $\beta=2.11$ for anterograde and $\alpha=2.76$ and $\beta=2.94$ for retrograde transport, respectively, suggesting a rather 
broad distribution of velocities (Figure 1G). Image-based simulations of IFT indicate that movement of eGFP-KIF13B inside cilia with such velocities over the entire cilia length of < $5 \mu \mathrm{m}$ would result in oscillation frequencies for bidirectional movement of about $30 \mathrm{sec}$ (Figure 2). The observed periodicity of intensity oscillations of eGFP-KIF13B in cilia was much slower; typically, between 200-500 sec (Fig. 1D and Movie 1,2). This dynamic behavior likely reflects the import and export kinetics of KIF13B in primary cilia combined with frequent switching of directions. It is comparable to the avalanche-like injection of IFT particles into cilia, observed previously (Ludington et al., 2013), indicating that eGFPKIF13B moves frequently up and down in cilia before eventually being exported/released from the cilia base or tip.

Quantitative live cell imaging analysis was additionally performed using motorless eGFP-KIF13B. This analysis demonstrated that motorless eGFP-KIF13B enters cilia in 7 out of 26 cells examined, but its fluorescence intensity was much lower in cilia than at the basal body, and bursts of intraciliary movement and ciliary tip release were not observed (Figure 3A, B). Hence intraciliary motility and ciliary tip release of KIF13B depend on its own motor domain. We could not detect eGFP-KIF13A in cilia of hTERT-RPE1 cells under similar conditions (Figure 3C), whereas kinesin-2 motor GFP-KIF17 was stably localized at the ciliary tip (Figure 3D), as expected (Dishinger et al., 2010).

A previous study indicated that KIF13B is not needed for EV release from cilia in mouse inner medullary collecting duct 3 (IMCD3) cells (Nager et al., 2017). Consistently, live imaging analysis of eGFP-KIF13B in ciliated IMCD3 cells stably expressing the ciliary membrane marker mCherry-Ar113b showed eGFP-KIF13B localization at the base of, but not within, cilia of these cells (Figure 3E). As cells lacking IFT27 contain increased ciliary levels of SMO (Eguether et al., 2014) and presumably also KIF13B (Mick et al., 2015), we wondered if the stable presence of SMO-tRFP in cilia of the hTERT-RPE1 used for our imaging analyses lead to the observed intraciliary movement and ciliary tip release of eGFPKIF13B. We therefore analysed if eGFP-KIF13B could enter cilia of IMCD3 cells, stably expressing the ciliary membrane marker mCherry-Arl13b, upon treatment with the SMO agonist purmorphamine. However, purmorphamine did not induce ciliary entrance of eGFPKIF13B in these cells (Figure 3F). This is in agreement with a recent cilia proteomics analysis that failed to detect KIF13B in cilia of Shh-stimulated IMCD3 cells (May et al., 2021), suggesting that ciliary entrance and movement of KIF13B is not triggered by the presence of SMO in cilia. 
In summary, our results demonstrate that KIF13B transiently enters cilia, moves bidirectionally within the organelle at speeds reminiscent of IFT, and occasionally undergoes EV-like release from the ciliary tip. Intraciliary movement and ciliary tip release require KIF13B's own motor domain and appears to be cell-type specific, as reported for KLP-6 in C. elegans (Morsci and Barr, 2011). Since KIF13B is a plus end-directed motor, its anterograde movement within cilia is likely powered by its own intrinsic motor activity, whereas its retrograde movement must be mediated via association of KIF13B with the dynein-2 driven retrograde IFT machinery. Supportively, a proteomics analysis indicated that KIF13B accumulates within cilia of Ift27 mutant IMCD3 cells (Mick et al., 2015), which are deficient in retrograde IFT- and BBSome-mediated ciliary export of membrane proteins (Eguether et al., 2014), indicating that KIF13B could be transported out of cilia by IFT and the BBSome. However, further work is required to determine how KIF13B intraciliary movement is coordinated with the IFT/BBSome machinery, and how KIF13B entrance into cilia is regulated.

Interestingly, we observed that SMO-tRFP was not co-released with eGFP-KIF13B at the ciliary tip, suggesting that the released eGFP-KIF13B-containing material/EV-like particles are generated from a subdomain of the ciliary membrane. The cholesterol-binding protein CAV1 was implicated in EV release in other contexts (Ni et al., 2020), and we speculate that EVs may be released from a subdomain of the ciliary membrane enriched in sterols and CAV1. If this indeed is the case, the ciliary accumulation of CAV1 observed in hTERT-RPE1 cells lacking KIF13B (Schou et al., 2017) could be a consequence of impaired release of CAV1-containing EVs from cilia. We are currently investigating this possibility.

\section{Materials and methods}

\section{Plasmids}

The following previously described plasmids were used: eGFP-KIF13B (Asaba et al., 2003), motorless eGFP-KIF13B (Lamason et al., 2010), eGFP-KIF13A (Schou et al., 2017), GFPKIF17 (Jaulin and Kreitzer, 2010), and pcDNA3.1 (+) (Thermo Fisher Scientific). Plasmids were propagated in Eschericia coli DH10B cells using standard procedures and purified using NucleoBond Xtra Midi EF Kit (Macherey-Nagel).

\section{Mammalian cell culture and transfection}


hTERT-RPE1 cells stably expressing SMO-tRFP were generously provided by Christopher J. Westlake and have been described previously (Lu et al., 2015). The cells were cultured in in Dulbecco's Modified Eagle Medium (DMEM; Thermo Fisher Scientific) supplemented with $10 \%$ heat inactivated fetal bovine serum (FBS; Thermo Fisher Scientific), 1\% penicillinstreptomycin (Thermo Fisher Scientific), and $10 \mu \mathrm{g} / \mathrm{mL}$ Hygromycin B. Mouse IMCD3 cells stably expressing mCherry-ARL13B were a generous gift from Peter Gorilak and Vladimir Varga at Laboratory of Cell Motility, Institute of Molecular Genetics of the Czech Academy of Sciences, Prague, Czech Republic. These were cultured in DMEM/F12 medium (Thermo Fischer Scientific) containing 10\% heat inactivated FBS and 1\% penicillin-streptomycin. For the imaging experiments, cells were seeded on glass-bottom dishes 35/22 mm (HBSt-3522) from Willco Wells. Cells were transfected with with KIF-encoding plasmid and empty vector pcDNA3.1 (+) (1 $\mu$ g total DNA) using $2 \mu$ l Lipofectamine 3000 Reagent and $2 \mu 1$ P3000 (Thermo Fisher Scientific). hTERT-RPE1 cells stably expressing SMO-tRFP were transfected a day after seeding and IMCD3 cells expressing mCherry-Arl13b were reverse transfected at the time of seeding. To induce ciliogenesis, cells were cultured overnight (16 hr) in a medium free of FBS and antibiotics. For hTERT-RPE1 cells, serum starvation started $2 \mathrm{hr}$ post transfection and for IMCD3 cells ca. $16 \mathrm{hr}$ post transfection. To activate the Shh pathway and promote SMO ciliary entrance in IMCD3 cells, the cells were serum starved with DMEM/F12 medium containing $2 \mu \mathrm{M}$ purmorphamine (Sigma, cat\# SML0868).

\section{Fluorescent live cell imaging}

Live cell imaging was carried out on several confocal microscopes, all temperature $\left(37^{\circ} \mathrm{C}\right)$ and $\mathrm{CO}_{2}(5 \%)$ regulated in a humidity chamber. These included a fully motorized Olympus IX83 inverted microscope equipped with a spinning disc (Yokogawa) and a Hamatsu ORCAFlash 4.0 digital camera (C13440); a motorized inverted Nikon-Andor spinning disk microscope equipped with CSU-X1 spinning disk (Yokogawa), and/or laser launcher, Okolab microscope stage incubator, and a perfect focus system (PFS) (at DaMBIC, University of Southern Denmark). Both 60x and 100x Numerical Aperture (NA) 1.4 oil objectives (Olympus) were used. The 488 and 561 laser lines were used for imaging eGFP and tRFP, respectively. The time-lapse sequences were obtained with time intervals ranging from 5 to $10 \mathrm{sec}$. For laser scanning confocal microscopy at DaMBIC, University of Southern Denmark, a Nikon Ti-2, A1 LFO confocal microscope with a Plan Apo $\lambda 100$ x NA 1.4 oil 
objective with the following excitation/emission settings was used: 488/525 nm for eGFP and $561 / 595 \mathrm{~nm}$ for tRFP. The interval for the time-lapse sequences was $3 \mathrm{sec}$ or $1.1 \mathrm{sec}$. All live cell imaging was carried out in DMEM or M1 medium containing $150 \mathrm{mM} \mathrm{NaCl}_{2}, 5$ $\mathrm{mM} \mathrm{KCL}, 1 \mathrm{mM} \mathrm{CaCl} 2,1 \mathrm{mM} \mathrm{MgCl} 2,5 \mathrm{mM}$ glucose, and $20 \mathrm{mM}$ HEPES.

\section{Image analysis and simulations}

All image analysis and simulations were carried out in ImageJ (https://imagej.nih.gov/ij/), either using existing plugins, or implemented as self-developed Macro scripts (available from the authors upon request). Subsequent time series analysis was implemented as Jupyter notebooks in Python (https://jupyter.org/). First, cilia intensity of eGFP-tagged KIF13B (wilttype or motorless mutant) was isolated based on the red fluorescent marker protein SMOtRFP. For that, a rectangular region of interest (ROI) was defined around the red cilia marker, and an intensity threshold was applied to the SMO-tRFP fluorescence in cilia weighted by the mean intensity of the red marker in the entire ROI. This procedure accounted for eventual photobleaching and secured a stable cilia area segmentation. A binary mask comprising the cilia area with intensities of either 0 or 1 was generated and multiplied with the corresponding green image series of the eGFP-tagged KIF13B. Integrated fluorescence intensity of eGFP-KIF13B was measured from videos with long acquisition time (i.e. 5-6 sec, acquired at a spinning disk confocal system) and normalized to the total green fluorescence in the rectangular ROI. To determine periodicities in cilia location dynamics of eGFP-KIF13B, the extracted intensity time series was transformed into frequency space using the Fast Fourier transform function of the scipy library in Python. Time-lapse sequences with shorter acquisition time (i.e. 1.1.sec intervals) were treated identically, but in addition to calculating integrated intensities, a Kymograph analysis was carried out. For that, the segmented cilia were first spatially aligned using a rigid body registration procedure. A 3-pixel wide line (straight or segmented) was used and the kymograph calculated using the Multikymograph plugin to Image J developed by Drs. A.Seitz and J. Rietdorf. Straight lines were identified and velocities of eGFP-KIF13B were calculated using the accompanying Macro script based on the known pixel size and interval time. Cumulative histograms of velocities measured in the anterograde and retrograde direction were calculated and fitted to a Weibull function as described in the Results and discussion section. Image simulations of cilia movement were implemented as ImageJ Macro with a fixed cilia length of $5 \mu \mathrm{m}$, a simulated pixel size of either 0.05 or $0.1 \mu \mathrm{m}$ and a frame rate of 1 frame per sec $(1 \mathrm{~Hz})$. A variable number of 
consecutive motors was translated towards the cilia tip with a constant speed. A stochastic velocity component was added from a uniform distribution, comprising about $0-10 \%$ of the total velocity to account for the stochastic nature of motor movement. Simulated images were convoluted with a Gaussian filter of width equal to 0.15 or $0.25 \mu \mathrm{m}$ and Gaussian noise was added to account for the blurring by the microscope optics and the camera read out noise, respectively.

\section{Acknowledgements}

We thank Drs Christopher J. Westlake, Valdimir Varga, Athar Chisthi, Joel Pomerantz and Geri Kreitzer for reagents. We thank Søren Lek Johansen for assistance with plasmid preparation. This study was supported by grants from the Novo Nordisk Foundation (NNF18OC0053024, NNF15OC0016886 and NNF14OC0011535), the Danish Cancer Society (R146-A9590) and Brødrene Hartmanns Fond (A31662) to LBP. JB was supported by an Eramus+ traineeship from the European Commision. DW acknowledges funding from the Villum foundation (Grant No. 35865). 


\section{Figure legends}

Figure 1. Transient bidirectional movement and EV-like tip release of eGFP-KIF13B in primary cilia of hTERT-RPE1 cells. (A) eGFP-KIF13B (green) is transiently moving into cilia, marked with SMO-tRFP (red). In less than one minute, eGFP-KIF13B accumulates in cilia and some is released from the cilia tip (arrows) between 360-400 sec of the video sequence. (B) Measurement of eGFP-KIF13B fluorescence intensity in cilia over time showing entry into cilia in repeated bursts. The video sequence in (A) is part of a longer recording, and the time window is indicated with a blue box. (C) Kymograph of the first part of sequence in (A) demonstrating that eGFP-KIF13B accumulation within cilia with this time resolution looks like two waves. (D) Fourier transformation (power spectrum) of the data in (B) with main periodicities indicated. (E) Example of similar analysis as in (B) with only one burst of eGFP-KIF13B observed (under the graph are example snapshots aligned with the curve). (F) Kymograph of high-time resolution sequences showing lines for anterograde (straight lines) and retrograde (dashed lines) eGFP-KIF13B intraciliary movement. (G) Weibull distribution function of the form $y(v)=1-\exp \left[-(\alpha \cdot v)^{\beta}\right]$ with $\alpha=1.58$ and $\beta=2.11$ for anterograde and $\alpha=2.76$ and $\beta=2.94$ for retrograde transport, respectively, suggests a rather broad distribution of eGFP-KIF13B velocities in hTERT-RPE1 SMO-tRFP cells.

Figure 2. Image simulations of cilia movement. (A) Left panel shows five simulated motors as consecutive single pixels and right panel shows the appearance of those motors through the microscope. (B) Kymographs calculated for images of one or 3 motors were used for validation of the analysis procedure for a nominal velocity of $0.25 \mu \mathrm{m} / \mathrm{sec}$. The true velocity can be recovered with $>95 \%$ accuracy. (C) Integrated intensity was quantified for a portion of the simulated cilia to emulate time-resolved measurements of eGFP-KIF13B in cilia (compare with Figure 1B and E). There were two periodic intensity peaks, since the quantification of cilia intensity allowed for partial exit of the simulated motors at the cilia base. (D) Fourier transform of the intensity profile reveals this 2-fold periodicity, the higher frequency corresponds to one-way transport and the lower frequency (given the periodicity of $30 \mathrm{sec}$ ) corresponds to the full bidirectional movement of the particles.

Figure 3. Control experiments for eGFP-KIF13B live cell imaging analyses. (A) Example snapshots of movie showing motorless eGFP-KIF13B in SMO-tRFP cells, which is mainly accumulating around the ciliary base with a fraction localizing stably to cilia over time (25 
cells imaged in total). (B) Measurement of motorless eGFP-KIF13B fluorescence intensity in cilia over time, based on movies as in (A). (C, D) Frames of representative movies from live cell imaging analysis of eGFP-KIF13A (C) and GFP-KIF17 (D) in hTERT-RPE1 SMO-tRFP cells. A total of 4 and 3 transfected cells were imaged for eGFP-KIF13A and GFP-KIF17, respectively. (E, F) Frames of movies from live cell imaging of eGFP-KIF13B in IMCD3 cells stably expressing mCherry-Arl13b (red) with no Hedgehog stimulation. (E, F) Representative time frame snap shots of eGFP-KIF13B in IMCD3 cells stably expressing mCherry-Arl13b (red) in the absence (E) or presence $(\mathrm{F})$ of $2 \mu \mathrm{M}$ purmorphamine. In these cells eGFP-KIF13B is localized at the two centrioles at the base of the cilia, marked by mCherry-Arl13b. A total of 10 and 16 cells were imaged for conditions in (E) and (F), respectively.

Movie 1. Live cell imaging movie showing transient, burst-like intraciliary movement and tip release of eGFP-KIF13B in RPE1 SMO-tRFP cells.

Movie 2. Additional live cell imaging movie showing transient, burst-like intraciliary movement and tip release of eGFP-KIF13B in RPE1 SMO-tRFP cells. 


\section{References}

AKELLA, J. S. \& BARR, M. M. 2021. The tubulin code specializes neuronal cilia for extracellular vesicle release. Dev Neurobiol, 81, 231-252.

AKELLA, J. S., CARTER, S. P., NGUYEN, K. C., TSIROPOULOU, S., MORAN, A. L., SILVA, M., RIZVI, F., KENNEDY, B. N., HALL, D. H., BARR, M. M. \& BLACQUE, O. E. 2020. Ciliary Rab28 and the BBSome negatively regulate extracellular vesicle shedding. eLife, 9, e50580.

ANVARIAN, Z., MYKYTYN, K., MUKHOPADHYAY, S., PEDERSEN, L. B. \& CHRISTENSEN, S. T. 2019. Cellular signalling by primary cilia in development, organ function and disease. Nat Rev Nephrol, 15, 199-219.

ASABA, N., HANADA, T., TAKEUCHI, A. \& CHISHTI, A. H. 2003. Direct Interaction with a Kinesin-related Motor Mediates Transport of Mammalian Discs Large Tumor Suppressor Homologue in Epithelial Cells. Journal of Biological Chemistry, 278, 8395-8400.

DISHINGER, J. F., KEE, H. L., JENKINS, P. M., FAN, S., HURD, T. W., HAMMOND, J. W., TRUONG, Y. N., MARGOLIS, B., MARTENS, J. R. \& VERHEY, K. J. 2010. Ciliary entry of the kinesin-2 motor KIF17 is regulated by importin-beta2 and RanGTP. Nat Cell Biol, 12, 703-10.

EGUETHER, T., SAN AGUSTIN, J. T., KEADY, B. T., JONASSEN, J. A., LIANG, Y., FRANCIS, R., TOBITA, K., JOHNSON, C. A., ABDELHAMED, Z. A., LO, C. W. \& PAZOUR, G. J. 2014. IFT27 links the BBSome to IFT for maintenance of the ciliary signaling compartment. Dev Cell, 31, 279-90.

GARCIA-GONZALO, F. R. \& REITER, J. F. 2017. Open Sesame: How Transition Fibers and the Transition Zone Control Ciliary Composition. Cold Spring Harb Perspect Biol, 9.

JAULIN, F. \& KREITZER, G. 2010. KIF17 stabilizes microtubules and contributes to epithelial morphogenesis by acting at MT plus ends with EB1 and APC. J Cell Biol, 190, 443-460.

LAMASON, R. L., KUPFER, A. \& POMERANTZ, J. L. 2010. The dynamic distribution of CARD11 at the immunological synapse is regulated by the inhibitory kinesin GAKIN. Mol Cell, 40, 798-809.

LECHTRECK, K. F. 2015. IFT-Cargo Interactions and Protein Transport in Cilia. Trends Biochem Sci, 40, 765-778.

LU, Q., INSINNA, C., OTT, C., STAUFFER, J., PINTADO, P. A., RAHAJENG, J., BAXA, U., WALIA, V., CUENCA, A., HWANG, Y. S., DAAR, I. O., LOPES, S., LIPPINCOTT-SCHWARTZ, J., JACKSON, P. K., CAPLAN, S. \& WESTLAKE, C. J. 2015. Early steps in primary cilium assembly require EHD1/EHD3-dependent ciliary vesicle formation. Nat Cell Biol, 17, 228-240.

LUDINGTON, W. B., WEMMER, K. A., LECHTRECK, K. F., WITMAN, G. B. \& MARSHALL, W. F. 2013. Avalanche-like behavior in ciliary import. Proc Natl Acad Sci USA, 110, 3925-3930.

LUXMI, R., KUMAR, D., MAINS, R. E., KING, S. M. \& EIPPER, B. A. 2019. Cilia-based peptidergic signaling. PLoS Biol, 17, e3000566.

MAY, E. A., KALOCSAY, M., D'AURIAC, I. G., SCHUSTER, P. S., GYGI, S. P., NACHURY, M. V. \& MICHK, D. U. 2021. Time-resolved proteomics profiling of the ciliary Hedgehog response. J Cell Biol, 220, e202007207. 
MICK, D. U., RODRIGUES, R. B., LEIB, R. D., ADAMS, C. M., CHIEN, A. S., GYGI, S. P. \& NACHURY, M. V. 2015. Proteomics of Primary Cilia by Proximity Labeling. Dev Cell, 35, 497-512.

MORSCI, N. S. \& BARR, M. M. 2011. Kinesin-3 KLP-6 regulates intraflagellar transport in male-specific cilia of Caenorhabditis elegans. Curr Biol, 21, 1-6.

NAGER, A. R., GOLDSTEIN, J. S., HERRANZ-PEREZ, V., PORTRAN, D., YE, F., GARCIA-VERDUGO, J. M. \& NACHURY, M. V. 2017. An Actin Network Dispatches Ciliary GPCRs into Extracellular Vesicles to Modulate Signaling. Cell, 168, 252-263 e14.

NI, K., WANG, C., CARNINO, J. M. \& JIN, Y. 2020. The evolving role of Caveolin-1: a critical regulator of extracellular vesicles. Med Sci (Basel), 8, 46.

PEDERSEN, L. B. \& ROSENBAUM, J. L. 2008. Intraflagellar transport (IFT) role in ciliary assembly, resorption and signalling. Curr Top Dev Biol, 85, 23-61.

PHUA, S. C., CHIBA, S., SUZUKI, M., SU, E., ROBERSON, E. C., PUSAPATI, G. V., SETOU, M., ROHATGI, R., REITER, J. F., IKEGAMI, K. \& INOUE, T. 2017. Dynamic Remodeling of Membrane Composition Drives Cell Cycle through Primary Cilia Excision. Cell, 168, 264-279 e15.

SCHOU, K. B., MOGENSEN, J. B., MORTHORST, S. K., NIELSEN, B. S., ALELIUNAITE, A., SERRA-MARQUES, A., FURSTENBERG, N., SAUNIER, S., BIZET, A. A., VELAND, I. R., AKHMANOVA, A., CHRISTENSEN, S. T. \& PEDERSEN, L. B. 2017. KIF13B establishes a CAV1-enriched microdomain at the ciliary transition zone to promote Sonic hedgehog signalling. Nat Commun, 8, 14177.

SERRA-MARQUES, A., MARTIN, M., KATRUKHA, E. A., GRIGORIEV, I., PEETERS, C. A., LIU, Q., HOOIKAAS, P. J., YAO, Y., SOLIANOVA, V., SMAL, I., PEDERSEN, L. B., MEIJERING, E., KAPITEIN, L. C. \& AKHMANOVA, A. 2020. Concerted action of kinesins KIF5B and KIF13B promotes efficient secretory vesicle transport to microtubule plus ends. Elife, 9, e61302.

TASCHNER, M. \& LORENTZEN, E. 2016. The Intraflagellar Transport Machinery. Cold Spring Harb Perspect Biol, 8.

WANG, J., SILVA, M., HAAS, LEONARD A., MORSCI, NATALIA S., NGUYEN, KEN C. Q., HALL, DAVID H. \& BARR, MAUREEN M. 2014. C. elegans Ciliated Sensory Neurons Release Extracellular Vesicles that Function in Animal Communication. Curr Biol, 24, 519-525.

WILLIAMS, C. L., MCINTYRE, J. C., NORRIS, S. R., JENKINS, P. M., ZHANG, L., PEI, Q., VERHEY, K. J. \& MARTENS, J. R. 2014. Direct evidence for BBSomeassociated intraflagellar transport reveals distinct properties of native mammalian cilia. Nat Commun, 5, 5813.

WOOD, C. R., HUANG, K., DIENER, D. R. \& ROSENBAUM, J. L. 2013. The cilium secretes bioactive ectosomes. Curr Biol, 23, 906-11.

WOOD, C. R. \& ROSENBAUM, J. L. 2015. Ciliary ectosomes: transmissions from the cell's antenna. Trends Cell Biol, 25, 276-85. 
Figure 1

bioRxiv preprint doi: https://doi.org/10.1101/2021.07.09.451768; this version posted August 16, 2021. The copyright holder for this preprint (which was not certified by peer review) is the author/funder. All rights reserved. No reuse allowed without permission.

A

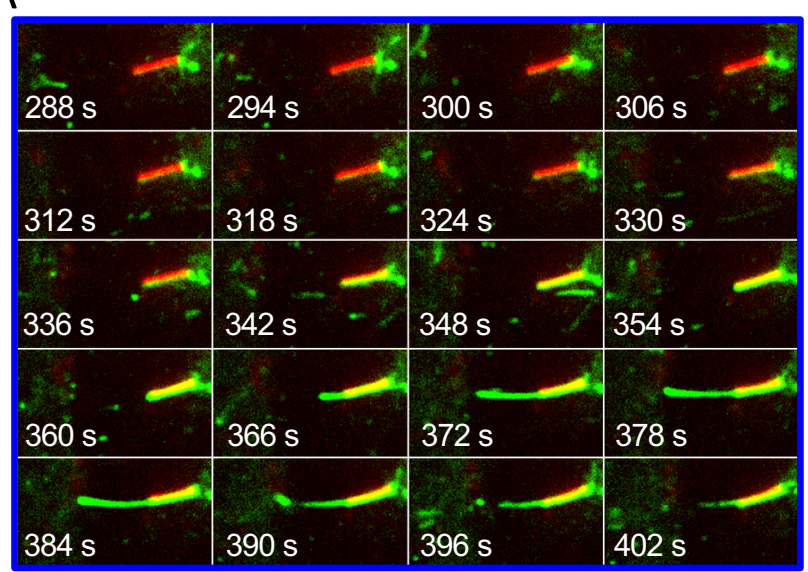

B Intensity of eGFP-KIF13B in cilia

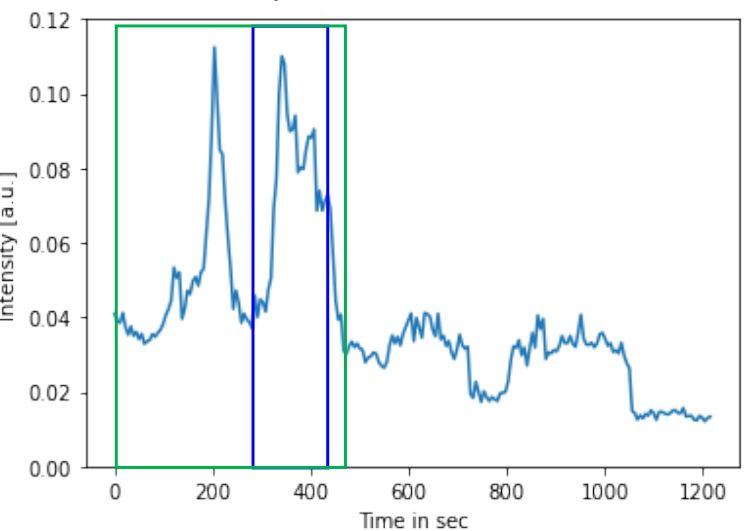

\section{C}
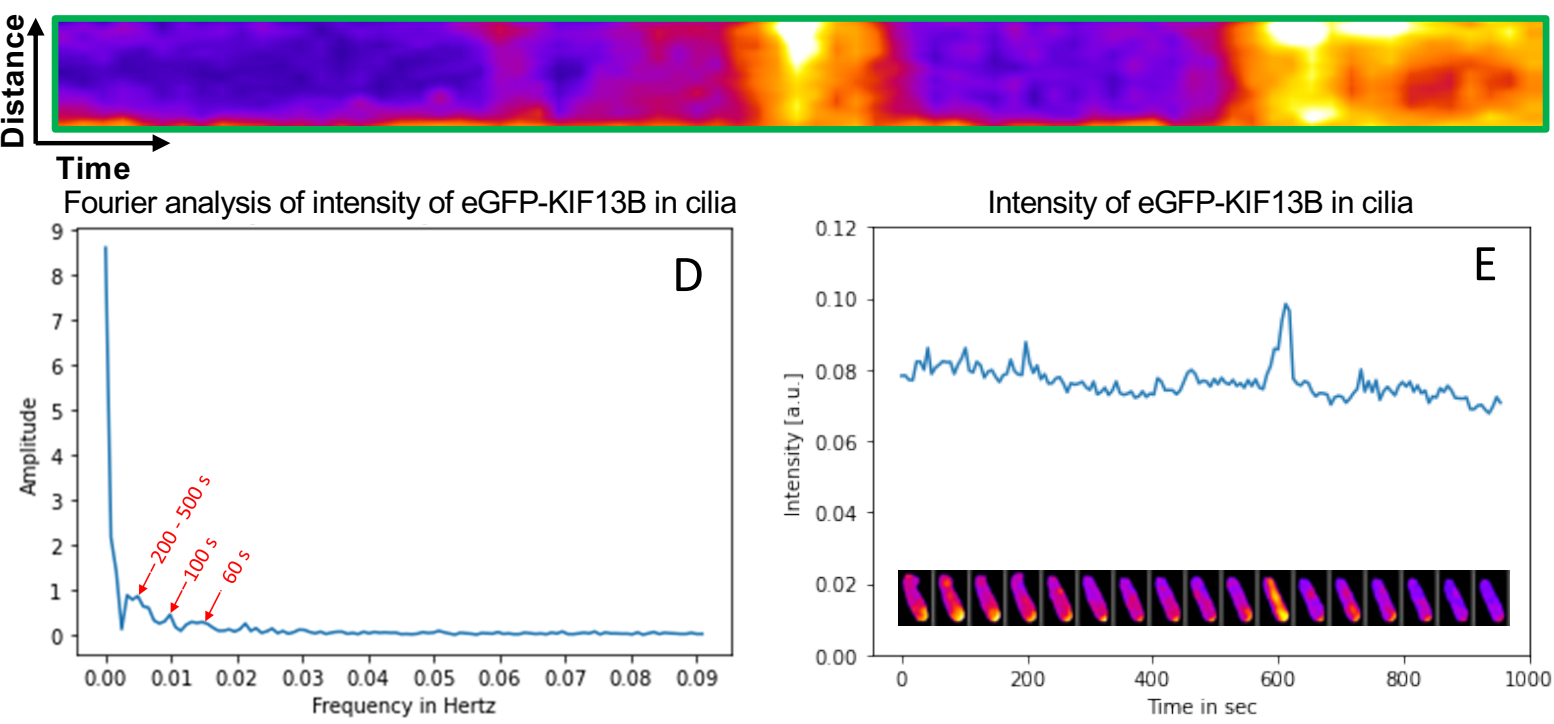

$\mathrm{F}$

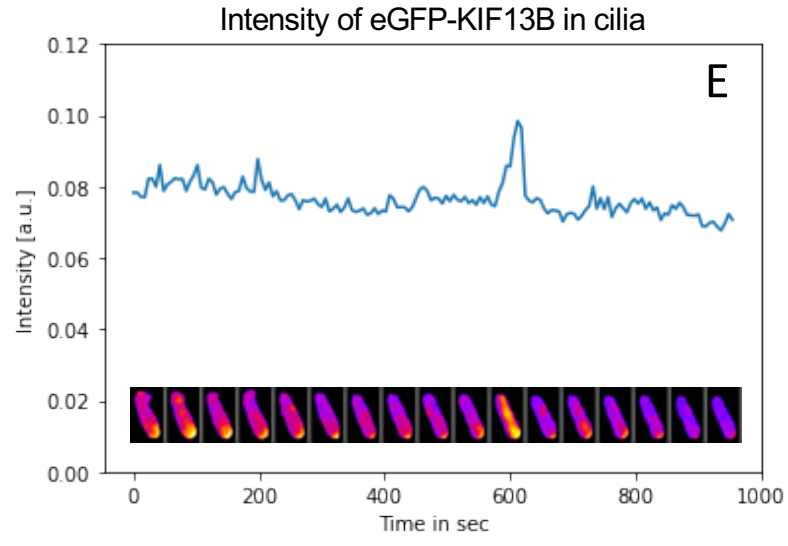

B

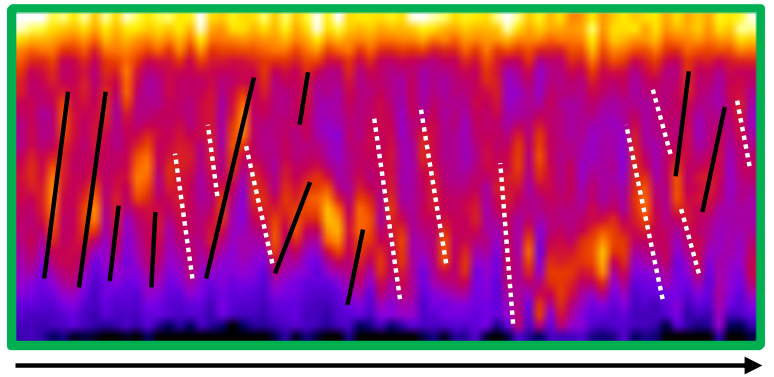

Time $=77 \mathrm{sec}$

G

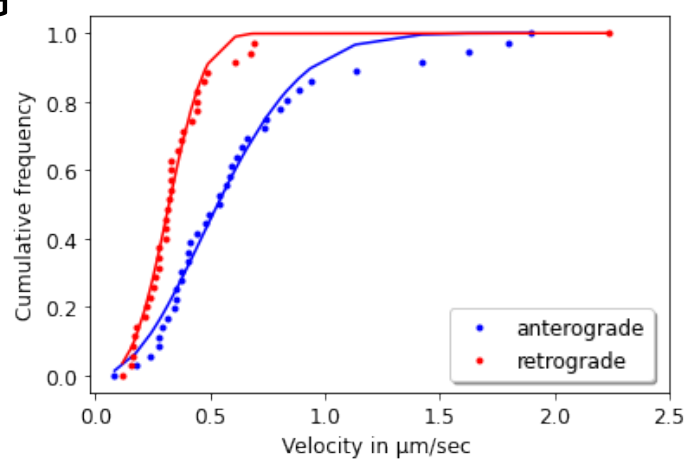


Figure 2

bioRxiv preprint doi: https://doi.org/10.1101/2021.07.09.451768; this version posted August 16, 2021. The copyright holder for this preprint (which was not certified by peer review) is the author/funder. All rights reserved. No reuse allowed without permission.

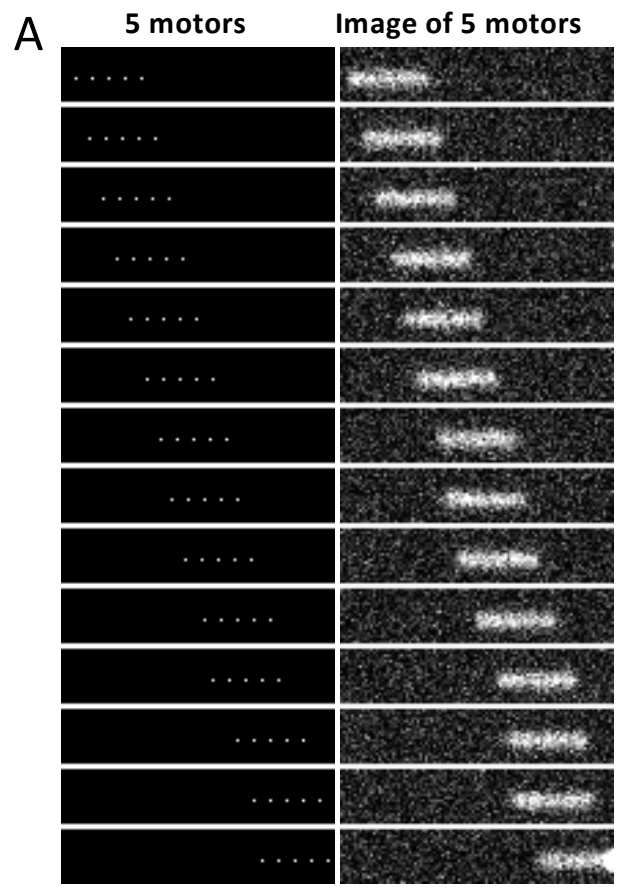

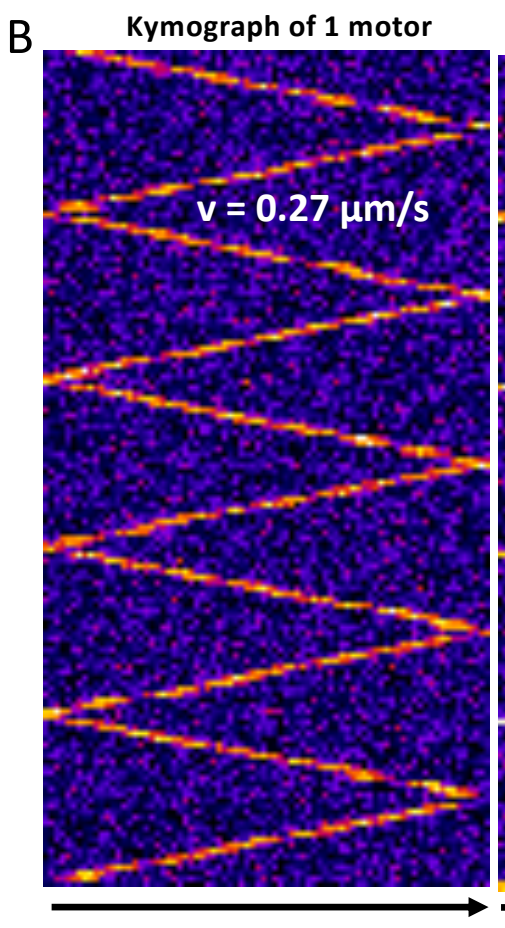

$4.5 \mu \mathrm{m}$
Kymograph of 3 motors

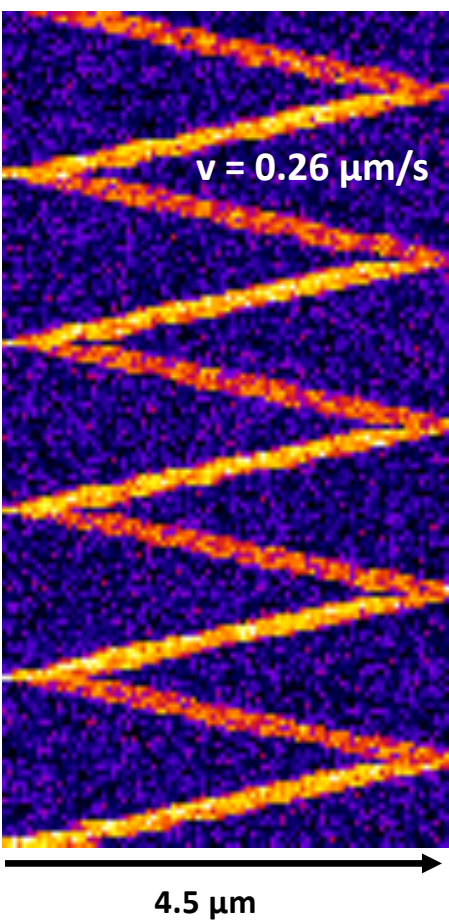

C

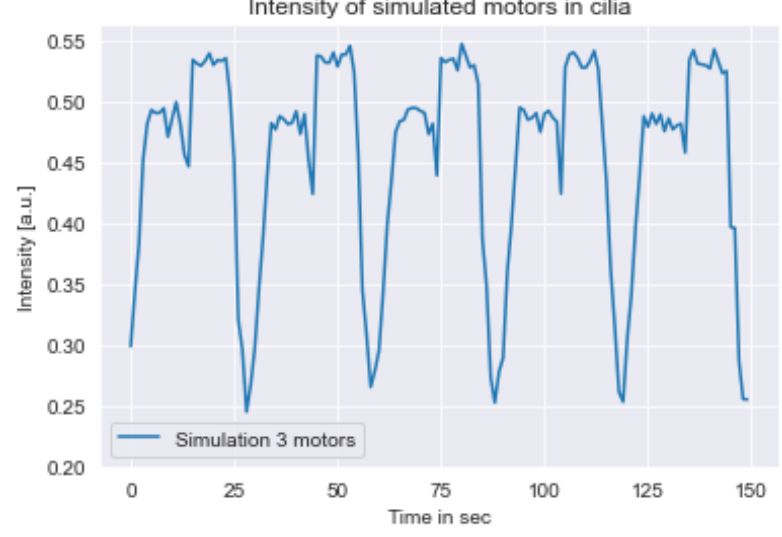

D

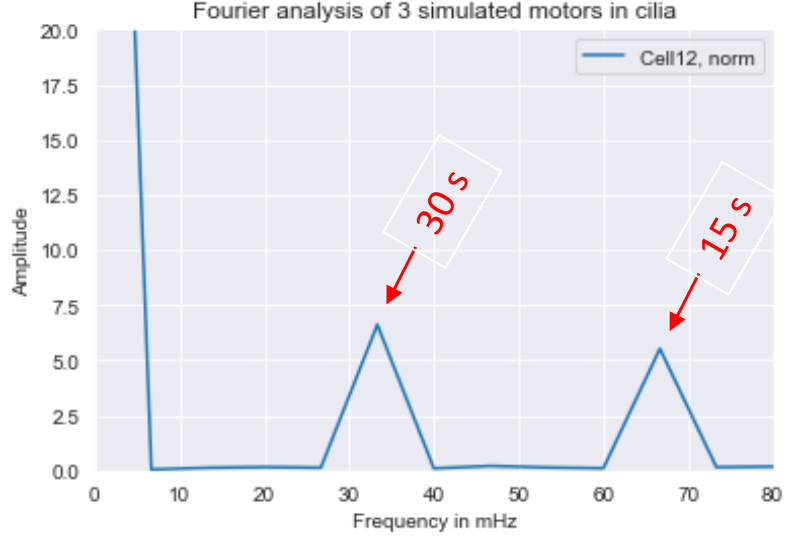




\section{Figure 3}

bioRxiv preprint doi: https://doi.org/10.1101/2021.07.09.451768; this version posted August 16, 2021. The copyright holder for this preprint (which was not certified by peer review) is the author/funder. All rights reserved. No reuse allowed without permission.

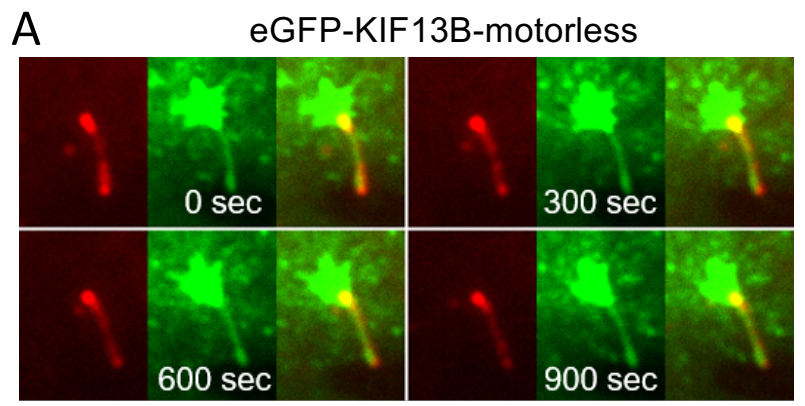

Intensity of eGFP-KIF13B-motorless in cilia

C
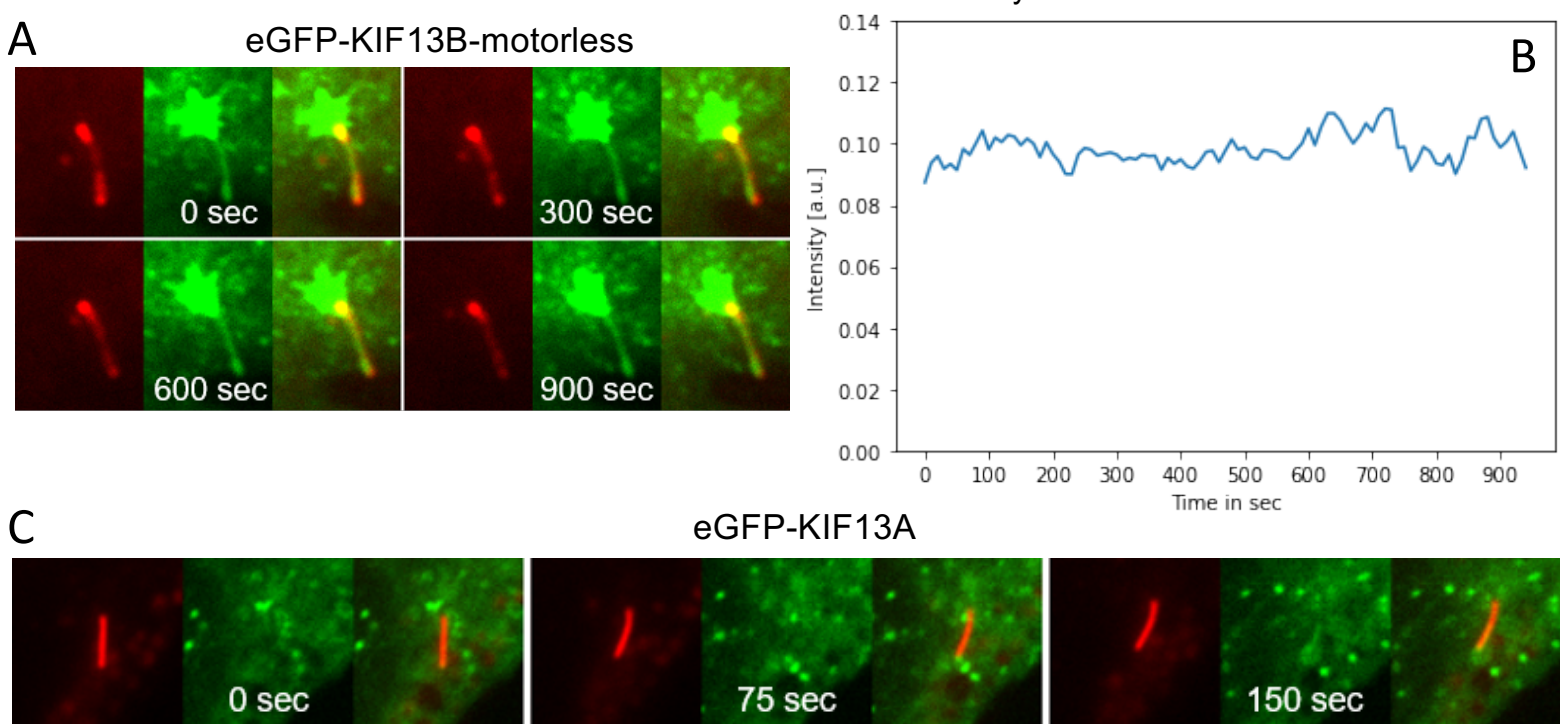

eGFP-KIF13A
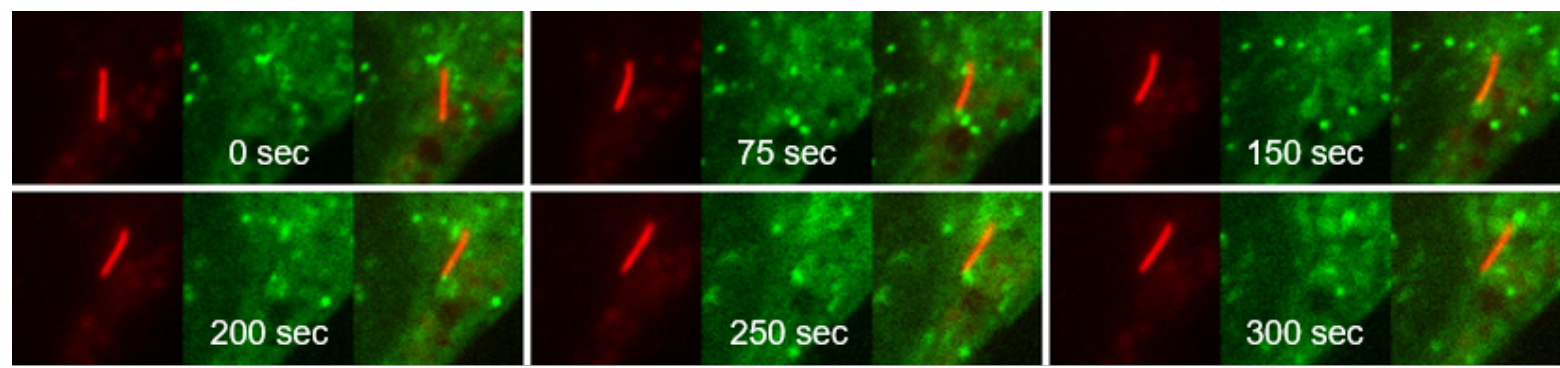

D

eGFP-KIF17
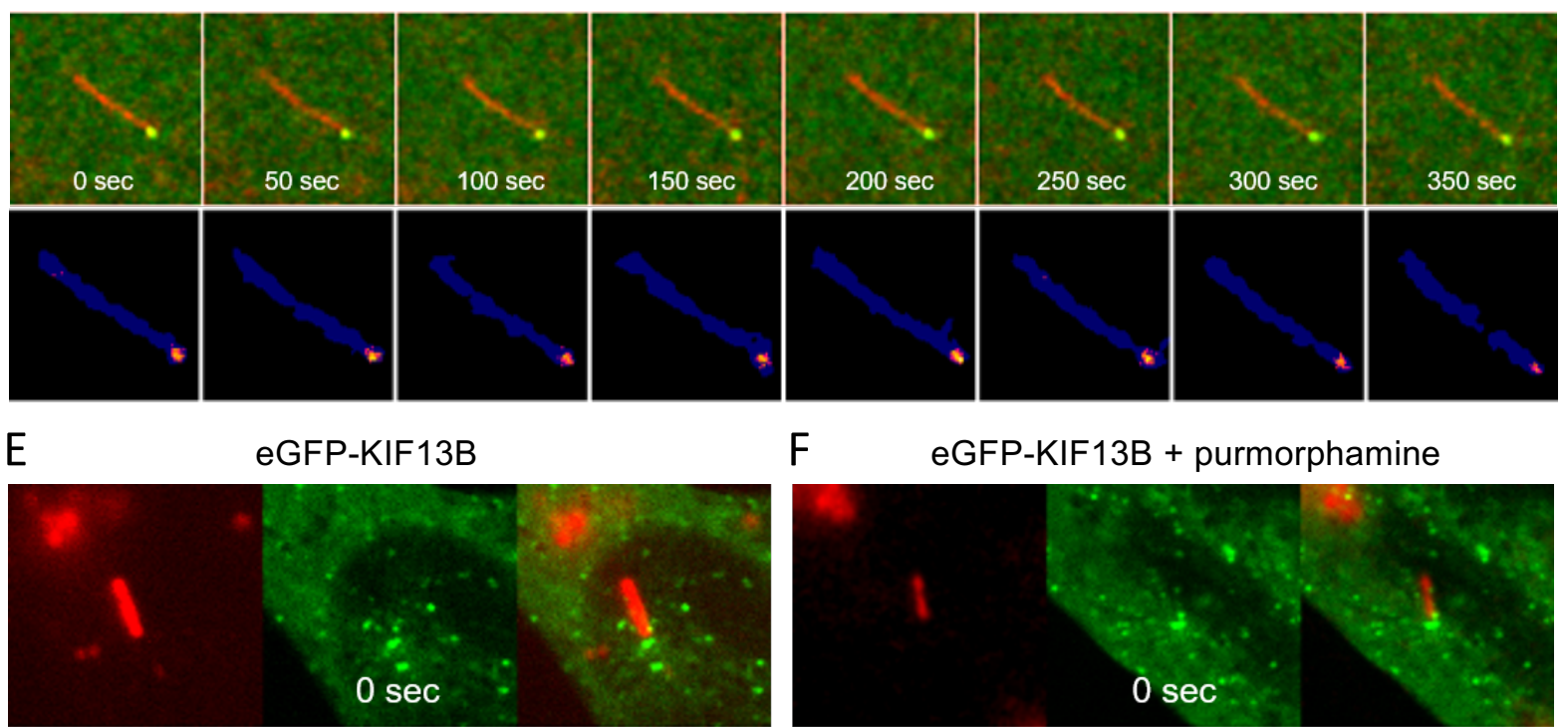

$\mathrm{F}$

eGFP-KIF13B + purmorphamine
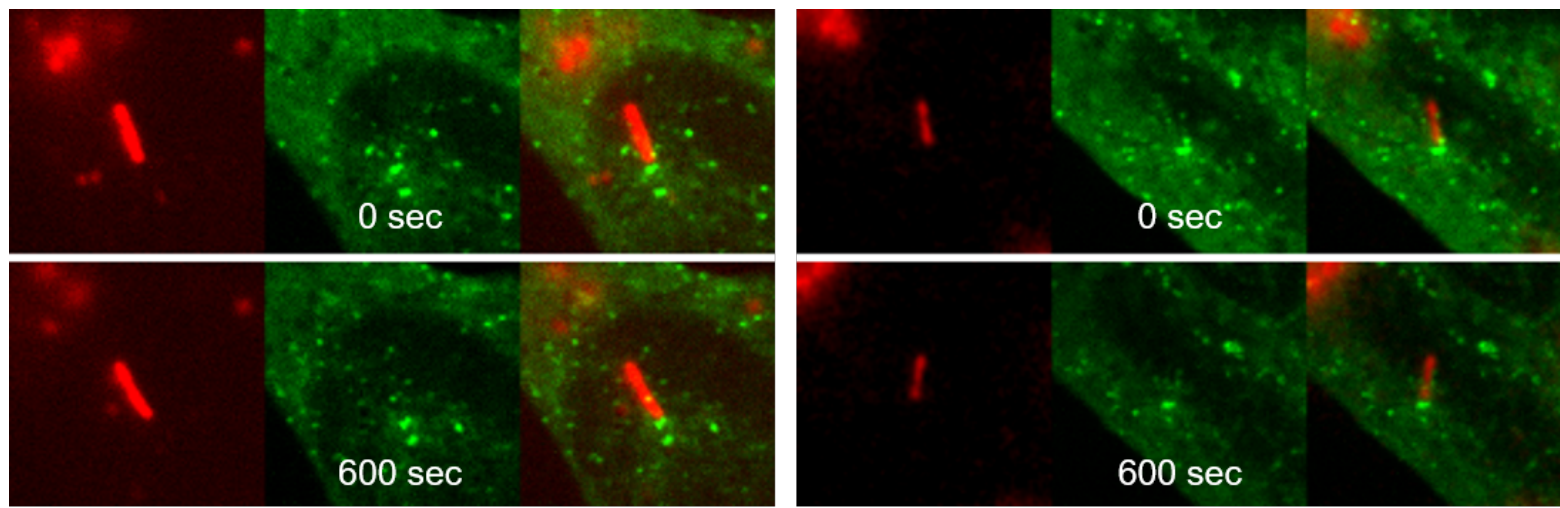